\title{
CORDOTOMIA PERCUTANEA
}

\author{
NELSON PIRES FERREIRA * \\ NEY A. V. DE CASTRO AZAMBUJA **
}

A interrupção cirúrgica dos feixes espinotalâmicos, a nivel medular, tem sido um procedimento de largo uso no tratamento da dor desde a sua primeira realização por Martin 3,23, em 1911. Diversas modificações foram agregadas à técnica inicial na tentativa de obter melhores resultados cirúrgicos.

Mullan \& col.1s iniciaram nova abordagem do problema, procedendo à destruição do quadrante antero-lateral medular com agulha radioativa, mediante punção do canal vertebral a nível cervical. Os mesmos autores 15 em 1965, já utilizando a punção lateral do canal, realizaram a lesão medular com corrente anódica. No mesmo ano, Rosomoff \& col.22, acrescentaram, como fonte de lesão, a radiofrequência. $O$ introdutor dessa técnica em nosso meio foi $\mathrm{J}$. Guelmann ${ }^{6}$, em 1973.

Após os relatos iniciais diversos autores informaram os seus resultados $1,4,5,8,9,10,11,12,13,14,15,18,20,21,24,26,27,28$. A presente comunicação estuda a experiência inicial com o método, em 47 procedimentos realizados.

\section{CASUISTICA E METODO}

$\mathrm{Em}$ um periodo de aproximadamente 4 anos, foram realizadas 47 cordotomias, em 43 pacientes; em 4, o procedimento fol realizado bilateralmente, em dois tempos, com intervalo de 10 a 25 dias. A média de idade dos pacientes foi de 49,6 anos, sendo o mais jovem de 17 e, o mais idoso, de 73 anos. O maior contingente de pacientes situou-se na $4^{\text {a }}$ e décadas de vida, 34 casos. Do total, 26 pacientes eram do sexo feminino a 17 do sexo masculino. A duração dos sintomas fol a seguinte: até 2 meses, 8 casos; 3 a 4 meses, 11 casos; 5 a 12 meses, 14 casos; mais de 1 ano, 10 casos.

A dor, nos casos estudados, tínha as seguintes caracteristicas: radicular, 10 casos; superficial e/ou latejante, 7 casos e, nos demais, profunda. Em 17 pacientes foi identifićada deficiencia sensitiva prévia ou queixa de disestesias em zonas cutaneas relacionadas com a dor. A topografia da dor, considerando o nível de projeçá ou de irradiação, teve a seguinte distribuição: lombar e/ou sacra, 26 casos; torácica 7; cervical e lombar 1 e cervico-torácica 1. Do total de 43 pacientes, 38 apresentavam neoplasia maligna e 5 dor de etíologia benigna, sendo 1 caso de nevralgia pos-herpética

Trabalho realizado no Instituto de Neurocirurgia de Porto Alegre; * ProfessorAdjunto e Livre-Docente de Neurocirurgia da Universidade Federal do Rio Grande do Sul; ** Neurocirurgiăo. 
de topografia torácica. Nos 2 primeiros casos fol tentada a realizaçăo da cordotomia, sem sucesso. Esses casos foram excluidos do presente estudo.

Todos os pacientes foram submetidos a cordotomia percutanea cervical ao nivel do espaço C1-C2. A preparaçăo consistiu em meticulosa explicaçăo do procedimento e sedação com Benzodiazepinona pré-operatoriamente ou com Inoval, 30 a $60 \mathrm{mg}$, endovenoso, imediatamente antes da punçăo.

O paciente é colocado em decabito dorsal horizontal com a cabeça imobilizada pelo fixajor de Rosomoff 22, que é previamente fixado na mesa do craniógrafo de Lysholm ou em mesa com cabeceira radiotransparente. O cranio é posicionado, em relacăo ao tórax, de tal modo que a porçăo alta da coluna cervical fique no plano horizontal. A punçăo é realizada com agulha 14/10, com bisel curto. $O$ local da punça se situa a $1 \mathrm{~cm}$ dorsal e caudalmente ao processo mastóideo. Antes da introduçáo da agulha o local é cuidadosamente infiltrado com anestésico. Além do referencia anatómica para a escolha do local da punçáo, estudo radiológico simples prévio, em perfil, ou controle com amplificador de imagem foram utilizados com o intuito de obter maior precisăo. A seguir, a agulha é introduzida com a mesma técnica da punção lombar. O curso da agulha, quando usado o intensificador de imagem, pode ser acompanhado dando maior segurança em seu deslocamento. $O$ alvo é, inicialmente, a zona equatorial do canal. Uma vez alcançado o espaço subaracnóideo, retira-se, em uma seringa, $3 \mathrm{ml}$ de liquido cefalorraqueano que é emulsionado com 2-3ml de Pantopaque ou Lipiodol e $2 \mathrm{ml}$ de ar.

Durante a introduçăo da agulha o paciente geralmente refere dor quando a mesma ultrapassa a dura-mater e quando a raiz C2 é traumatizada. Fm continuaça a agulha é fixada em um sistema que permite o deslocamento nos tré planos. A relaça da agulha, em seu plano antero-posterior, com o ligamento denticulado, é verificada pela realização de mielografia mediante a injeçáo da emulstio previamente preparada. As imagens da mielografia podem ser seguidas no intensificador de imagens ou em estudos radiológicas repetidos da região cervical, em perfil (Fig. $1, B$ e C).

A mielografia permite a identificaçăo da face anterior da medula e/ou ligamento denticulado e/ou da face posterior da medula (Fig. 1, B e C). A extremidade da agulha, na projeçăo de perfil, deve ficar situada a $2 \mathrm{~mm}$ posteriormente à face anterior da medula ou a $2 \mathrm{~mm}$ anteriormente ao ligamento dentículado. Após a localizaçáo correta da agulha, nesse plano, estudo radiologico em antero-posterior, transoral, permite a colocaçáo da extremidade da agulha em um plano que passe na base do odontóide (Fig. 1 A). Ap6s a localizaçăo correta da agulha é introduzido um eletrodo de aco inoxidável de $0,4 \mathrm{~mm}$, apontado com eletrólise e isolado por manguito de polietileno até $2-3 \mathrm{~mm}$ de sua extremidade, em substituiçăo ao mandril. Esse eletrodo deve ultrapassar em 4-5 mm a extremidade da agulha-canula. A extremidade năo isolada do eletrodo penetra na medula e o polietlleno serve de degrau evitando que o eletrodo penetre na medula, além de sua porçăo descoberta. O eletrodo é conectado ao polo ativo do gerador de radiofrequencia (modelo $2 \mathrm{AV}$-Radionic) e a agulha-canula é conectada com o polo indiferente. Nease momento é iniciada a estimulaçăo com a frequência de $5 \mathrm{~Hz}$ e $1-3 \mathrm{~V}$ e, a seguir, $70-100 \mathrm{~Hz}$ e $0,2-0,8 \mathrm{~V}$. Os estimulos, com essas caracteristicas, motivam as seguintes respostas: 5Hz e 1-3V - contracho ipsolateral dos músculos do pescogo, espectalmente o trapério, eventualmente sensagoes contralaterale; 70-100Fs e 
0,2-0,8V - sensações contralaterais de calor, frio, queimadura, agulhadas ou dormência. A lesăo não é feita quando ocorrerem abalos motores nos segmentos ipsolaterais, na presença de sensações ipso ou bilaterais e quando năo houver resposta sensitiva contralateral.

Após a obtenção da resposta desejada, sensações contralaterais, quer no membro superior, tronco ou membro inferior, procede-se à lesão. A lesão é feita, em etapas, pelo aquecimento do eletrodo utilizando uma voltagem de $20 \mathrm{~V}$ durante 10, 15, 20, 25 e 30 s, em sequência, com estudo dos resultados após cada lesão. Esse estudo consiste no exame da motricidade, ipsolateral à cordotomia, e das diversas formas de sensibilidade, no dimídio contra lateral. Os niveis de analgesia e eventualmente hipoestesia, usando etapas progressivas de lesão, atingem o membro inferior, tronco e membro superior e pescoço. Na dependência da topografia do eletrodo intramedular, pode ocorrer deficiência de sensibilidade com curso inverso, isto é, iniciando-se no membro superior, progredindo caudalmente. O procedimento é dado por concluido quando é

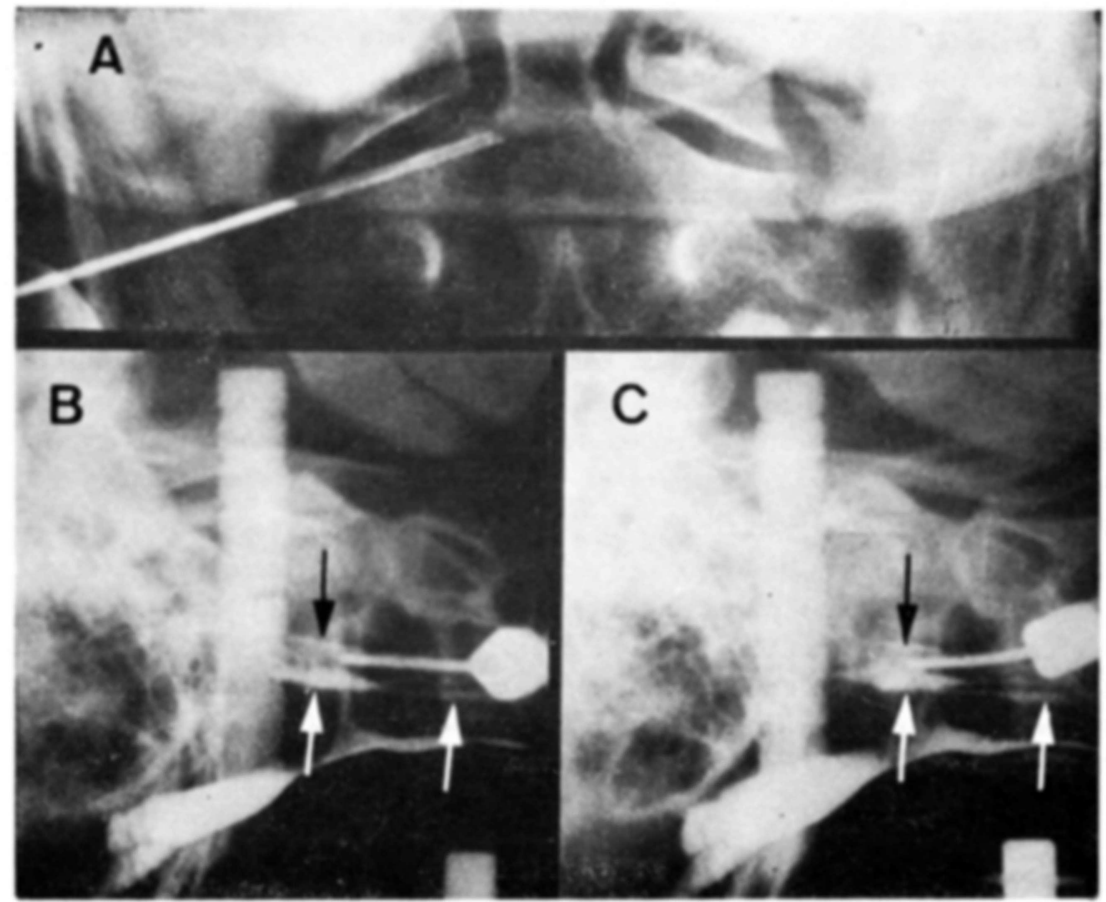

F'ig. 1 - Controle radiologico em AP, transoral (A); extremidade da agulha no plano da base do odonitoide. Controle radiologico em perfil - mielografia - identificaç̃o da superficie anterior da medula (seta preta) $e$ do ligamento denticulado (sotas grancas) (B e C). Em $B$ a extremidade da agulha esta muito anterior; em $C$, após elevaço do canhão da agulha, houve posicionamento correto da sua extremidade no plano $A P$, em relação ao ligamento denticulado. 
alcançado o nivel de analgesia pretendido. Logo após o procedimento é administrada Dexametasona, $20 \mathrm{mg}$ EV, que é mantida nos três dias subsequentes, na dose de $4 \mathrm{mg} \mathrm{HV}$, cada 6 horas.

\section{RESULTADOS}

Em 47 cordotomias realizadas os niveis de analgesia obtidos foram: C2, 20 casos; C3-C4, 3 casos; T1, 1 caso; T3-T4, 16 casos; T5-T6, 2 casos e T10-T12, 3 casos. Nos quatro pacientes submetidos a cordotomias bilaterais os niveis obtidos foram: C3-T4, 2 casos; T3-T4, 1 caso e T4-T5, 1 caso. Fm um paciente não foi obtida analgesia quer superficial, quer profunda, havendo, entretanto, desaparecimento da dor que motivou o procedimento até a alta hospitalar.

Deficiência motora ipsolateral ocorreu em 7 casos; em 3 durante o procedimento e, em 1, no dia subsequente; nos três restantes, a deficiência foi identificada na sala de recuperação. Em 1 caso ocorreu hemiparesia nítida, havendo melhora nas dias subsequentes, persistindo hemiparesia que não interferia com a atividade da paciente. Em 10 casos foi observada ataxia ipsolateral que persistiu por 5 a 21 dias. Retenção urinária transitória foi identificada em três casos, sendo em 1, após a segunda cordotomia, no lado oposto. Apnéia de sono ocorreu em 1 caso, complicação que motivou o obito do paciente, apesar das medidas pertinentes. Sindrome de Claude BernardHorner, ipsolateral à lesăo, é um achado frequente e está relacionada com a altura da cordotomia.

Cinco pacientes, submetidos a cordotomia para tratamento de dor unilateral, passaram a referir dor contralateral, com as mesmas caracteristicas ou diversas da dor inicial, no pós-procedimento imediato. Dois pacientes, com patologia benigna, passaram a referir disestesias nas áreas de analgesia, consideradas desconfortáveis, meses após a cordotomia. Em 1 paciente houve recurrência da dor, quatro meses após. Em 2 pacientes não houve controle completo da dor, havendo melhora dos sintomas, persistindo a necessidade de analgésicos.

O acompanhamento mais longo, em patologia maligna, correspondeu a 2 anos. A paciente tínha metćstases de carcinoma de mama, ao nível da fossa supraclavicular, como causa da dor Irradiada para o membro superior. Essa paciente, na ultima avaliaçã், mantinha nivel de hipalgesia em C4, com áreas de analgesia sem distribuiçăo metamérica. Vários pacientes não foram seguidos por diversas razóes, fato que dificulta uma análise dos resultados longinguos.

\section{COMENTARIOS}

A técnica inicial de Mullan \& col.15 que consistia na utilização de agulha com estrôncio ${ }^{90}$, apesar dos resultados satisfatórios, apresentava inconvenientes; a destruição era progressiva e evoluia até três meses após o procedimento. Esse fato impossibilitava a obtenção imediata de uma lesão que controlasse uma dor localizada no membro superior, sem risco de comprometimento motor ipsolateral. Com a utilização de corrente anódica, os mesmos autores ${ }^{16}$ obti- 
veram analgesia em um tempo variável de 2 a 10 minutos de corrente. Em alguns casos foi necessária a utilização da corrente por 30 minutos, fato que prolongava o procedimento. Esses autores ${ }^{15}$, na comunicação inicial, já preconizavam a estimulação medular e comentavam as respostas, considerando-a importante na indicação da topografia intramedular da extremidade do eletrodo. Com a introdução da radiofrequência, como fonte de lesão, Rosomoff \& col.22 abreviaram o tempo de lesão para períodos variáveis de 10 a 30 s.

Mullan \& col. ${ }^{15}$ preconizam a fixação da cabeça em um apoio em forma de $U$, no qual não há imobilidade e a localização do eletrodo intramedular é feita a mão livre. Rosomoff $\&$ col. ${ }^{22}$ preferem a melhor fixação possível da cabeça, em fixador por eles idealizado; a localização e as variações da posição do eletrodo são feitas mediante a manipulação dos cursores micrométricos, aos quais está fixada a aste que apoia a agulha-cânula. Utilizamos a última técnica por nos parecer mais segura; ela evita movimentos intempestivos do paciente durante sensações de dor e os deslocamentos da agulha e/ou do eletrodo podem ser medidos nos parafusos micrométricos aferidos.

As referências anatômicas adotadas para a realização da punção são as mesmas propostas por Mullan \& col. 16 aproximadamente $1 \mathrm{~cm}$ caudal e dorsalmente à extremidade do processo mastóideo. Essas referências são importantes quando é utilizado equipamento radiológico convencional. Quando da utilização de amplificador de imagem e televisão, a verificação do local da punção e o deslocamento da agulha é mais preciso, na incidência de perfil, face a possibilidade da observação continuada.

Os reparos anatomorradiológicos, para a escolha da posição da extremidade da agulha no canal raqueano, são a face anterior da medula e o ligamento denticulado. As suas identificações podem ser feitas mediante contraste positivo - Pantopaque ou Lipiodol - e a da superfície ventral da medula, mediante contraste positivo e/ou ar. Na casuística em estudo foram utilizados, na maioria dos casos, os contrastes positivos na demarcação dos acidentes anatômicos. Naqueles em que foi utilizado ar, como meio de contraste, ocorreram vômitos e queixa de dor, por vezes desconfortável, o que motivou o seu abandono.

A face lateral do processo odontóideo corresponde à margem lateral da dura-mater. A extremidade da agulha deve ficar, aproximadamente, em um plano que passe na base do odontóide ou a 5-6mm da linha média. A distância entre o ligamento denticulado e a borda interna da lâmina de C2 não excede a $10 \mathrm{~mm}{ }^{24}$. Onofrio 19 refere, como distância média, a de 7,5 mm. Para esse autor o ligamento denticulado está, em média, a $11 \mathrm{~mm}$ da borda posterior do corpo de C2. Para fins práticos, a abordagem do canal, em primeira instância, deve ser em sua zona equatorial. Essa posição inicial permitiria, devido a localização anterior ao ligamento, o deslocamento anterior ou posterior da extremidade da agulha-cânula e/ou do eletrodo, quando o canhão da mesma é deslocado no sentido postero-anterior. $O$ ponto ideal de penetração do eletrodo na medula é $2 \mathrm{~mm}$ posteriormente à superfície anterior da medula ou a $2 \mathrm{~mm}$ anteriormente ao ligamento. Essa posição é confirmada com o estudo mielográfico. A utilização da pneumorraque, com a finalidade de delimitar a super- 
fície anterior da medula, desencadeia dor que, frequentemente, motiva movimentos inconvenientes por parte do paciente.

Mullan 13 localiza o eletrodo a $1 \mathrm{~mm}$ anteriormente ao ligamento denticulado, quando trata dor de topografia lombossacra, e a $2 \mathrm{~mm}$, quando a dor é de topografia cervical. O mesmo autor 13 prefere introduzir, na medula, $3-4 \mathrm{~mm}$ do eletrodo quando a dor é cervical e, menos de $3 \mathrm{~mm}$, quando a dor é no membro inferior. Nas situações em que a dor é perineal, Mullan 13 sugere a localização do eletrodo posteriormente ao ligamento denticulado. Lesões situadas no quadrante posterior da medula foram identificadas por Rosomoff em estudos pósmortem sem que as mesmas desencadeassem deficiência motora. É difícil precisar a extensão exata da penetração do eletrodo intramedular face a grande mobilidade da medula. Nos parece que o melhor reparo é a extensão descoberta do eletrodo; sua cobertura de polietileno serviria como degrau limitador da penetração.

Face a área restrita da medula que deve ser lesada, as variações anatômicas, a proximidade dos feixes de projeção com funções motoras próximos ao espinotalâmico e a imprecisão relativa dos métodos radiológicos, especialmente devida à mobilidade medular a nível cervical, é absolutamente necessária a confirmação topográfica do eletrodo mediante estimulação do feixe espinotalâmico antes da realização da lesão. Os estudos de Rakic \& Yakovlev, referidos por Sweet 24, comprovam a grande variação na sintopia das fibras longas a nível medular. Esses achados explicam e alertam para os possíveis insucessos e complicações advindas de lesão anatômica no quadrante anterior da medula. Autores como Rosomoff \& col.22 e Onófrio ${ }^{19}$ não se utilizam desse elemento como reparo na adequação da topografia da extremidade do eletrodo. Taren \& col. ${ }^{25}$ obtiveram, com estímulos de $1-6 \mathrm{~Hz}$ e $0,3-1,5 \mathrm{~V}$, resposta motora do trapézio ipsolateral, devido a estimulação do corno anterior e de fibras motoras ventrais da raiz C2. Respostas caracterizadas por movimentos sincrônicos dos dedos, deltóide e dos músculos do membro inferior indicam que o eletrodo está próximo ao feixe cortico-espinal. Estimulação com frequências de $60-100 \mathrm{~Hz}$ e $0,25-3 \mathrm{~V}$ motivam respostas sensitivas contralaterais referidas, pelo paciente, como sensação de frio, calor, queimadura, agulhadas ou formigamento. Quando as respostas sensitivas contralaterais ocorrem com a intensidade de $0,25 \mathrm{~V}$, a extremidade do eletrodo está localizada no ponto de maior concentração de fibras. Quando as respostas contralaterais ocorrem com $0,5 \mathrm{~V}$, a extremidade do eletrodo está próxima e, quando ocorrem com intensidade superior a $0,7 \mathrm{~V}$, é sinal que está distante. Nesse caso há necessidade de relocalização da extremidade do eletrodo. Quando as respostas sensitivas são ipsolaterais à punção, ou bilaterais, especialmente nos membros inferiores, é indicativo que o eletrodo está situado no feixe de Goll e Burdach ou na região centromedular, fato que contraindica a realização da lesão. As sensações motivadas pela estimulação podem estar localizadas no membro superior, tórax e membro inferior. Segundo Tasker $\&$ col. ${ }^{26}$, quando há resposta sensitiva contralateral no membro superior, especialmente mão, ocorre analgesia de $\mathrm{C} 4$ para baixo, após a lesão. Quando há resposta sensitiva distal, a analgesia é também distal e, na maioria das vezes, ocorre paresia de membro inferior. Na nossa casuística, o maior contingente de pacientes 
referiu, durante a estimulação, disestesias no membro inferior. Em poucos casos, houve queixa de sensações localizadas no membro superior, não sendo possivel, entretanto, confirmar a assertiva de Tasker \& col..26.

Nos pacientes tratados sempre foi obtida a referência de sensações contralaterais, antes de ser feita a lesão. Nas situações em que houve resposta motora motora ou sensitiva ipsolateral, o eletrodo foi recolocado até ser obtida resposta adequada. Em alguns casos, apesar das referências radiológicas indicarem uma correta topografia, a estimulação não o confirmou. Esse fato fortalece a necessidade de estimulação como teste, antes da feitura da lesão.

As lesões podem ser realizadas mantendo a voltagem em $20 \mathrm{~V}$ e aumentando o tempo, em etapas de 5 segundos, de 10 a 30, como preferem Rosomoff \& col.22. Mullan ${ }^{14}$ prefere manter o tempo em 30 segundos, aumentando a milamperagem; inicia com $20 \mathrm{~mA}$ aumentando, gradativamente, em cada lesão, até atingir 50mA. Em ambas as situações, após cada lesão, as diversas formas de sensibilidade são testadas, especialmente a dolorosa, o mesmo ocorrendo com o estudo da motilidade. Segundo Taren \& col.25, que utilizam eletrodos não isolados em $3 \mathrm{~mm}$, as lesões têm $4-5 \mathrm{~mm}$ de comprimento e $2-3 \mathrm{~mm}$ de diâmetro, acompanhando $o$ eixo do eletrodo. A lesão completa se faz após 15-20 segundos, momento em que há queda da corrente verificada no painel do aparelho. Nos pacientes tratados foi utilizada à técnica de Rosomoff \& col. ${ }^{22}$, voltagem fixa, tempo variável.

A casuística estudada consta de 43 pacientes, sendo 26 do sexo feminino e 17 do sexo masculino. A predominância do sexo feminino decorre da origem dos pacientes de um serviço de ginecologia. O maior contingente esteve situado nas $4^{a}$ e $5^{a}$ décadas de vida, 34 casos. A duração dos sintomas dolorosos variou de um mês a mais de 1 ano, não sendo identificada diferença nos resultados em relação ao tempo de duração da dor. A característica da dor, radicular, superficial ou profunda, também não influenciou nos resultados. Em 17 casos havia, além da dor, fenômenos deficilitários sensitivos e/ou motores. Nesse grupo não foi verificada diferença nos resultados, em relação ao grupo sem deficiência prévia ao procedimento.

Os niveis de analgesia obtidos foram: cervicais 23 casos (49\%), em C2, 20 casos $(42,5 \%)$; torácico alto, 20 casos $(42,5 \%)$ e torácico baixo, 3 casos $(6,5 \%)$. Em 1 paciente não foi obtido nível sensitivo, persistindo a ausência da dor que motivou o procedimento até a alta hospitalar. Mullan 13 explica essa ocorrência pelo deslocamento posterior das fibras sensitivas a esse nível medular. A analgesia ocorre, por vezes, sem grande ou nenhum comprometimento da sensibilidade tactil; esse fato é relatado por Tasker \& col..26.

Os níveis de analgesia obtidos pelos diversos autores é muito variável, provavelmente na dependência das suas necessidades terapêuticas. Taker \& col.2i informam que os níveis obtidos são geralmente nos dermátomos C5-C6 e, ocasionalmente, em C4. Bettag \& col. 1 identificaram niveis cervicais em $50,1 \%$, torácico alto em $17,9 \%$ e torácico baixo em $32 \%$. Taren \& col.25 não obtiveram nível desejado em $20 \%$ dos seus casos. Rosomoff \& col..22 identificaram nível de analgesia cervical ou torácica alta em $60 \%$ de sua 
casuística; $30 \%$ desses pacientes näo necessitavam nível tão alto para o controle da dor. O mesmo autor verificou, na revisão de seus pacientes, que $54 \%$ deles tinham, três meses após o procedimento, o mesmo nível ou nível mais alto de analgesia (19\%), em $13 \%$ houve necessidade de repetição do procedimento devida a queda do nível; em $9 \%$, o nível desapareceu. Desses, apenas 4 necessitaram repetição da cordotomia.

Considerando a necessidade da obtenção de um nível de analgesia para controle da dor, relacionada com a sua topografia e possíveis quedas de nível longínquas ao procedimento, identificamos na casuística que: em 14 casos de dor cervical foram obtidos niveis em C2 (13 casos) e em C4 (1 caso), adequada às necessidades; em 7 casos de topografia torácica os níveis variaram de $\mathrm{C} 2-\mathrm{C} 4$ (5 casos) a T3 (1 caso) e sem analgesia, 1 caso, também considerados resultados adequados; em 26 casos de dor relacionada com os membros inferiores, foram identificados níveis convenientes em 21 casos (T3 a T12) e, em 5, superiores, face às necessidades, níveis de $\mathrm{C} 2$ a C3. Do total de 47 procedimentos consideramos niveis acima das necessidades em 5 pacientes, o que corresponde a 10,6\%. Em 2 pacientes não houve controle completo da dor, havendo melhora considerável $(4,3 \%)$. Face a perda do seguimento de boa parte dos pacientes foi impossível avaliar os resultados longíquos, bem como, as variações de nível no pós-procedimento distante. $\mathrm{Em} 1$ paciente houve apnéia de sono que motivou, indiretamente, o óbito $(2,1 \%)$. A revisão da literatura 8 indica uma mortalidade que varia de 0 a $4,6 \%$.

Deficiência motora transitória foi identificada em 7 pacientes (14,9\%) e pẹmanente em $1(2,1 \%)$; a literatura 8 informa, respectivamente, 4 a $17 \%$ e 0 a $3 \%$. Ataxia ipsolateral à lesão ocorreu, nos casos estudados, em 10 $(21,3 \%)$; os achados na literatura 8 estão em torno de $28 \%$. O desencadeamento de disfunção vesical foi observada em 2 casos $(4,3 \%)$, sendo em 1 , após a segunda cordotomia controlateral. Esse tipo de disfunção, pós-cordotomia, tem sido relatada 8 em um percentual que varia de 1.5 a $15 \%$.

Dos 47 procedimentos em apenas 2 casos $(4,2 \%)$ não houve controle completo da dor, havendo, entretanto, melhora considerável dos sintomas. Nos casos estudados foi conseguido controle imediato da dor em 95,7\% dos casos e benefício em $4,2 \%$. A experiência de diversos autores 8 informam resultados que oscilam entre 80 e $96 \%$.

A indicação de cordotomia bilateral cervical alta merece maior atenção face o risco de perturbações respiratórias que dela podem advir. Nem sempre há sinais premonitórios indicativos, durante o procedimento, de uma disfunção respiratória futura. Essa disfunção pode aparecer 4 a 5 dias após a cordotomia. Mullan ${ }^{13}$ não recomenda a sua realização quando o P02 está abaixo de $70 \mathrm{mmHg}$.

Segundo Nathan ${ }^{18}$, as fibras reticuloespinais, embora bilaterais, exercem um controle unilateral no homem. As lesões do feixe espinotalâmico acima de $\mathrm{C} 4$, em cordotomias altas, podem interromper essas fibras reticuloespinais, motivando disfunção respiratória: $\mathrm{O}$ mecanismo respiratório permanecendo intacto unilateralmente, é capaz de manter uma função respiratória satisfatória desde que não haja doença degenerativa crônica, pneumopatia aguda, extensa 
redução da área pulmonar por ressecção, paralisia do nervo frênico ou insuficiência cardíaca; situações isoladas ou em combinação ${ }^{17,27}$. A presença das patologias acima ref̣eridas contraindicam, formalmente, a realização de cordotomia alta pelo risco que o procedimento envolve. Em situações de dúvida ou de rotina, a opção pela técnica percutânea transdiscal de Lin \& col.7 ou a cordotomia aberta por via posterior abaixo de C5 ou por via anterior pela técnica de Cloward ${ }^{2}$, com destruição medular com bisturi ou radiofrequência, devem ser consideradas. A experiência de diversos autores é concorde que a cordotomia não é um procedimento ideal para o controle da dor em doenças benignas devido a incidência de disestesias frequentemente desconfortáveis. Essa ocorrência foi observada em 2 pacientes.

A avaliação da casuística permite concluir que o procedimento é de fácil realização, permitindo o controle da dor na maioria dos pacientes. E um procedimento pouco traumático que pode ser indicado em pacientes em mau estado geral. A sua realização, com o paciente acordado, oferece a vantagem da observação precisa dos resultados imediatos.

\section{RESUMO}

São estudados os resultados obtidos em 47 cordotomias percutâneas realizadas em 43 pacientes. A técnica utilizada, os reparos anatômicos, o controle radiológico e o neurofisiológico são descritos e comentados. Não foi observada diferença nos resultados tendo como referência a duração e o tipo de dor, bem como a existência prévia de deficiência sensitiva. As complicações decorrentes da lesão medular são descritas e comparadas com as relatadas na literatura, observando-se resultados semelhantes. $O$ estudo da casuística permite concluir que o método é útil, pouco traumático, podendo ser indicado em pacientes em mau estado geral. A realização da lesão, com o paciente acordado, oferece a vantagem de um controle mais preciso dos resultados imediatos.

\section{SUMMARY}

\section{Percutaneous cordotomies.}

The results obtained in 47 percutaneous cordotomies performed in 43 patients are reported. The methods used, the anatomic references, the radiologic and neurophysiologic controls are described and commented. No diference was observed in the results if we consider the type and duration of the pre-existing pain, as well as the previous presence of sensory deficit. The benefits and complications of such form of treatment are described and compared with the ones presented in the literature, being equivalent. The study of this cases permit to conclude that this form of treatment is useful, causes little trauma and is well tolerated by patients in poor general conditions. The performance of the lesion with the patient awake adds the advantage of permitting a better control of the immediate results. 


\section{REFERENCIAS}

1. BETTAG, W.; WANDT, H. \& ROOSEN, K. - Pain treatment of adivanced malignant diseases by higth cervical percutaneous curdotomy. In Advances in Neurosurgery 3: Brain Hypoxia; Pain - Penzholz, H.; Brock, M.; Hamer, J.; Klinger, M. \& Spoerri, O., ed. Sprınger-Verıag, Berlin, p. 186-189, 1975.

2. CLOWARD, R. B. - Cervical cordotomy by the anterior approach. J. Neurosurg. $21: 19,1964$.

3. CRAWFORD, J. V. \& WALKER, A. E. - Surgery for pain. In A History of Neurological surgery - Walker, A. E., ed. Hafner Publishing, New York, cap. 13, p. 308-330, 1976.

4. ENTZIAN, W. \& LINKE, D. - Experience with percutaneous cordotomy. In Advances in Neurosurgery 3: Brain Hypoxia; Pain - Penzholz, H.; Brock, M.; Hamer, J.; Klinger, M. \& Spoerri, O., ed. Springer-Verlag, Berlin, p. 190-194, 1975.

5. FERREIRA, N. P.; AZAMbUJA, N. A. V. C. \& WAgNeR, R. C. - Cordotomia percutanea no tratamento da dor. Rev. AMRIGS (Porto Alegre) 20:235, 1976.

6. GUelmanN, J. M. - Comunicação pessoal.

7. LIN, P. M.; GILDENBERG, P. L. \& POLAKOFF, P. P. - An anterior approach to percutaneous lower cervical cordotomy. J. Neurosurg. 25:553, 1966.

8. LORENZ, R.; GRUMME, T.; HERMANN, D.; PALlESKE, H.; KUHNER, A.; STEUDE, U. \& ZIEURSKI, J. - Percutaneous cordotomy. In Advances in Neurasurgery 3: Brain Hypoxia; Pain - Penzholz, H.; Brock, M.; Hamer, J.; Klinger, M. \& Spoerri, O., ed. Springer-Verlag, Berlin, p. 178-185, 1975.

9. Mazars, G. - Atat actuel de la chirurgie de la douleur. Neuro-Chirurgie (Paris) 22, suppl 1:41, 1976.

10. MUKE, R. \& CORREIA, A. - Cordotomia percutanea cervical. Arq. Neuro-Psiquiat. (São Paulo) 32:15, 1974.

11. MUKE, R. \& CORREIA, A. - Potentials and limits of percutaneous cervical cordotomy. In Advances in Neurosurgery 3: Brain Hypoxia; Pain - Penzholz, H.; Brock, M.; Hamer, J.: Klinger. M. \& Spoerri. O., editors. Springer-Verlag, Berlin, p. 195, 1975.

12 MUllaN, J. F. - Percutaneous cordotomy - In Treatment of Pain - Voris. H. C. \& Whisler, W. W., ed. Charles C. Thomas, Springfield (Illinois) p. 75, 1975.

13. MUllaN, S. - Percutaneous cordotomy. J. Neurosurg. 35:360, 1971.

14. MUllaN. S. - Percutaneous cordotomy (R. F.) - In Advances in Neurology Bonica, J. J., ed. Raven Press Pub., New York, p. 677, 1974.

15. MULLAN, S.; HARPER, P. V.; HEKMATEANAH, J.; TORRES, H. \& DOBBEN, G. - Percutaneous interruption of spinal pain tracts by means of strontiun needle. J. Neurosurg. 20:931, 1963.

16. MUllan, S.: hekmateanah, J.; Dobben, G. \& BeCKMaN, F. - Percutaneous intramedulary cordotomy utilizing unipolar anodal electrolitic lesion. $J$. Neurosurg. 22:548, 1965.

17. MULLAN, S. \& HOSOBUCHI, Y. - Respiratory hazards of higth cervical percutaneous cordotomy. J. Neurosurg. 28:291, 1968. 
18. NATHAN, P. W. - The descending respiratory pathway in man. J. Neurol. Neurosurg. Psychiat. $26: 487,1963$.

19. ONOFRIO, B. M. - Cervical spinal cord and dentate delineation in percutaneous radiofrequency cordotomy at the level of the first to second cervical vertebrae. Surg. Gyn. Obstet. 133:30, 1971.

20. ROSOMOFF, H. L. - Bilateral percutaneous cervical radiofrequency cordotomy. J. Neurosurg. 31:41, 1969.

21. ROSOMOFF, H. L. - Percutaneous radiofrequency cervical cordotomy for intractable pain. In Advances in Neurology 4. - Bonica, J. J., ed. Raven Press Pub., New York, p. 683, 1974.

22. ROSOMOFF, H. L.; CARROL, F.; BROWN, J. \& SHEPTAK, P. - Percutaneous radiofrequency cervical cordotomy technique. J. Neurosurg. 23:639, 1965.

23. SPILLER, W. G. \& MARTIN, E. - The treatment of persistent pain of organic origin in the lower part of the body by division of the anterolateral column of the spinal cord. J. A. M. A. 20:1489, 1912.

24. SWEET, W. H. - Recent observations pertinent to improving anterolateral cordotomy. Clin. Neurosurg. 23:80, 1976.

25. TAREN, J. A.; DAVIS, R. \& CROSBY, E. C. - Target physiologic corroboration in stereotaxic cervical cordotomy. J. Neurosurg. 30:569, 1969.

26. TASKER, R. R.; ORGAN, L. W. \& SMITH, K. C. - Physiological guidlines for localization of lesions by percutaneous cordotomy. In Acta Neurochirurgia Advances in Stereotatic and Functional Neurosurgery - Guillingham, F. J.: Hitchcock, E. R. \& Turner, J. W., ed. Springer-Verlag. Berlin. Suppl. 21, p. 111. 1974.

27. TASKER, R. R. - Open cordotomy. In Progress in Neurological Surgery: Pain Its Neurological Management, Part II: Central Procedures - Krayenbuhl, H.: Maspes, P. E. \& Sweet, W. H., ed. S. Karger, Basel, vol. 8, p. 1-13. 1977.

28. WHITE, J. C. \& SWEET, W. H. - Pain and Neurosurgeon. Charles C. Thomas Pub., Springfield (Illinois), p. 678, 1969.

Instituto de Neurocirurgia - Pavilhão sđo José - Prą̧a Dom F'eliciano - 90000 Porto Alegre RS - Brasil. 